Toru Komatsu MD, Prabhat K. Singh MD, Tomomasa Kimura MD, Kimitoshi Nishiwaki MD, Kenji Bando MD, Yasuhiro Shimada MD

\section{Differential effects of ketamine and mida- zolam on heart rate variability}

of their opposing effects on autonomic nervous activity. However, normalized unit power showed the expected sympathetic activation with ketamine and sympathetic depression with midazolam since ketamine increased nuLF and midazolam decreased nuLF.

Il est possible de mesurer l'activité autonome initiée par l'anesthésie au moyen de lanalyse spectrale des fluctuations de la fréquence cardiaque (FFC). Cette étude recherche les effets de la kétamine et du midazolam sur les FFC. Trente patients ASA PS I font partie de l'étude. Quinze ont reçu de la kétamine $\left(2 \mathrm{mg} \cdot \mathrm{kg}^{-1}\right)$ et quinze du midazolam $\left(0,3 \mathrm{mg} \cdot \mathrm{kg}^{-1}\right) \dot{\mathrm{N}}$. Les intervalles $R R$ ont été mesurés à l'ECG avant et après linduction de l'anesthésie pendant dix minutes de respiration spontanée. La densité de la puissance spectrale des données a été calculée après transformation rapide sur une échelle de Fourier. Les pointes spectrales de chaque mesure ont été calculées: zone de basse fréquence $(L F, 0.04-0,15 \mathrm{~Hz})$, zone de haute fréquence ( $\mathrm{HF}, 0,15-0.5 \mathrm{~Hz})$ et puissance totale $\left(T P_{;} 0,04-0,5 \mathrm{~Hz}\right)$. L'unité de puissance normalisée a été dérivée comme suit: zone de basse fréquence (mLF): LF/TP $\times 100 \%$, zone de haule fréquence (nuHF): HF/TP $\times 100 \%$. La kétamine et le midazolam provoquent des réductions de toutes les mesures de puissance des FFC $(P<0,05)$. Cependant la kétamine a augmenté nuLF de $64 \pm 14 \%$ à $75 \pm 13 \%(P<0,05)$ et a diminué nuHF de $36 \pm 14 \%$ à $25 \pm 13 \%(P<0,05)$, alors que le midazolam a diminué nuLF de $66 \pm 14 \%$ à $54 \pm 14 \%(P$ $<0,05)$ et a augmenté nuHF de $34 \pm 15 \%$ à $46 \pm 14 \%(P$ $<0,05)$. Ces résultats montrent que la kétamine et le diazépam réduisent tous deux la puissance totale et toutes les compasantes fréquentielles de la puissance malgré leurs effets oppasés sur l'activité nerveuse autonome. Cependant, l'unité de puissance normalisée a montré, comme on s'y attendait, l'activation sympathique par la kétamine et la dépression sympathique par le midazolam étant donné que la kétamine augmentait nuLF et que le midazolam diminuait nuLF.

Beat-to-beat variations in heart rate (HR) reflect the dynamic responses of the cardiovascular control system to naturally occurring physiological perturbations. The autonomic nervous system (ANS) is an intricate feedback 
network with exquisitely sensitive capabilities of maintaining cardiovascular homeostasis. The rapid control of HR is effected primarily through the balance of vagal fibres and cardiac sympathetic fibres innervating the sinoatrial node. Power spectral analysis (PSA) of heart rate variability (HRV) provides frequency specific measures of oscillation amplitude which correlate with sympathetic and parasympathetic reflex activity. ${ }^{\prime}$

Different anaesthetics exert their different effects on the cardiovascular system by modulating ANS activity. Power spectral analysis of HRV may provide important clinical information on the influence of anaesthesia on the ANS and central nervous system (CNS), because cyclical variation in HR is mediated via a central neural mechanism as well as via baroreceptors and chemoreceptors. We reported that isoflurane decreased power of HRV in all frequencies in a dose-related fashion. ${ }^{2}$ We also noted a reduction in total power in myocardial revascularization patients who received fentanyl and diazepam. ${ }^{3}$ In addition, all other previous studies with thiopentone, propofol, and sufentanil, except etomidate, have shown reduction in all frequency components of HRV.,5 However, considerable differences may exist with different anaesthetics on frequency-specific effects of HRV. It was postulated that the profound decrease in HRV was associated with loss of consciousness but that normalized unit power of HRV (specific frequency fraction to total power) may still be a monitor of autonomic balance during anaesthesia. ${ }^{4}$

No study has been carried out to document the effects of ketamine and midazolam on PSA of HRV. Ketamine and midazolam are commonly used as iv anaesthetic induction agents. Ketamine is known to produce cardiovascular effects that resemble sympathetic nervous system stimulation. Direct stimulation of the CNS leading to increased sympathetic outflow seems to be the mechanism of the cardiovascular effects of ketamine. ${ }^{6}$ On the contrary, midazolam produces transient depression of baroreceptor mediated HR responses following intravenous administration in doses appropriate for induction of anaesthesia. ${ }^{7}$ A decrease in plasma norepinephrine concentration suggests a diminution of activity of the sympathetic efferent nerve pathway of the reflex. ${ }^{7}$

We investigated (1) whether both ketamine and midazolam, which have opposing cardiovascular effects, similarly decrease power of HRV after induction of anaesthesia and (2) whether changes in the balance of autonomic nervous activity caused by ketamine or midazolam are accompanied by changes in normalized unit power of HRV.

\section{Methods}

We studied 30 ASA physical status 1 patients scheduled for gynaecological or upper abdominal surgery. All patients were free from neurological, cardiac and pulmonary diseases, and no patient was receiving any chronic medication. After approval of the institutional review board, informed consent was obtained. Patients were studied before their scheduled surgical procedure started. Fifteen patients each were allocated randomly to receive ketamine $2 \mathrm{mg} \cdot \mathrm{kg}^{-1} \dot{w}$, or midazolam $0.3 \mathrm{mg} \cdot \mathrm{kg}^{-1} \dot{w}$ for induction of anaesthesia. Premedication consisted of hydroxyzine $0.1 \mathrm{mg} \cdot \mathrm{kg}^{-1}$ im given $45 \mathrm{~min}$ before the start of the study. On arrival in the operating room, patients were placed supine on a padded operating table and kept warm with a blanket while a peripheral $\dot{i}$ cannula was inserted under local anaesthesia. Ringer's lactate solution was infused throughout the rest of the study at a rate of 10 $\mathrm{ml} \cdot \mathrm{kg}^{-1} \cdot \mathrm{hr}^{-1}$. Tonometric blood pressure was monitored continuously using a Colin JENTOW (Nippon Colin, Komaki, Japan) on the radial artery. The electrocardiogram (ECG), and pulse oximetry were measured continuously throughout the period of study. The ECG lead representing the largest QRS amplitude at preoperative examination was chosen for recording. Before the start of anaesthesia, patients breathed $100 \%$ oxygen through a mask. On-line R-R intervals were measured ten minutes before induction of anaesthesia and thereafter measurements were continuously performed until ten minutes after induction and stabilization of heart rate. All patients were allowed to breath spontaneously $100 \%$ oxygen through mask. No attempt was made to assist or control respiration. Rectal temperature was measured at the end of the study.

Patients having a respiratory frequency between 0.2 to $0.4 \mathrm{~Hz}$ before induction of anaesthesia were selected for the subsequent study.

Data acquisition and data processing were performed by a specifically built mobile analysis system based on a personal computer (PC9801, NEC, Japan). On-line analysis of the $R-R$ intervals was made from the analogue signal of the ECG monitor by means of a hardwareimplemented R-wave detector (Nippon Colin, Komaki, Japan). This hardware filtered the ECG with a narrow band filter with centre frequency at $17 \mathrm{~Hz}$ and rectified the signal to obtain a signal merely existing of pronounced R-waves. This signal was fed into a signal threshold detector of which the threshold level automatically adjusted to $90 \%$ of the peak level and produced a rectangular pulse each time an $\mathbf{R}$ wave was detected. The resulting signal was then input into a universal counter board (UCM-4398BPC, Microscience, Tokyo, Japan) in a personal computer and calculated RR intervals were stored on a floppy disc. Off-line spectral analysis was performed on 128-sec segments of RR intervals. When present, extrasystolic beats were deleted and re- 
placed by an appropriately spaced value, and missing beats were inserted by interpolation. An instantaneous heart rate (IHR) time series was constructed as $1 / R R$ interval length and sampled at $4 \mathrm{~Hz}$ utilizing the method described by de Boer. ${ }^{8}$ A 100-point moving average was stepped through 512 segments of IHR time series. The template from this procedure was subtracted from the data set and provided residuals that were free of trends and slow sinusoidal changes. This procedure functions as a high-pass filter with a cutoff frequency of approximately $0.02 \mathrm{~Hz}$. The IHR signal was normalized by mean HR as described elsewhere. ${ }^{2}$ Then, spectral analysis of HRV was performed with fast Fourier transform (FFT). The power spectra, therefore, have the units of frequency ${ }^{-1}\left(\mathrm{~Hz}^{-1}\right)$. Power of HRV was quantified by determining the areas of the spectrum in two component widths: LF, 0.04-0.15 Hz; HF, 0.15-0.5 Hz. Total spectral power (TP, 0.04-0.5 Hz) was obtained as a sum of LF and HF. The normalized unit power of spectral peaks within each measurement were calculated as follows: nuHF, HF/TP $\times 100(\%)$, was considered as an indicator of parasympathetic activity; ${ }^{9}$ nuLF, LF/TP $\times 100(\%)$, was taken as an indicator of sympathetic activity or "sympathovagal balance." 9

Trended measurements of HRV were derived by sequential analysis of overlapping 128-sec data segments, with each sequential 128-sec segment beginning $32 \mathrm{sec}$ after the beginning of the previous segment. Example results are shown in Figure 1. The sequential HRV spectra are plotted as a three-dimensional surface that describes changes in HRV power over time.

Preinduction values of HRV were obtained by averaging the eight sequential trended HRV measurements obtained just prior to induction (corresponding to a 352sec time period). The maximal effect of the induction drugs on trended HRV measurements usually occurred between 90 and $180 \mathrm{sec}$ after drug administration and was of variable duration. The postinduction measurements used for comparison with preinduction measurements were obtained by averaging eight sequential trended measurements during the period shortly after maximal reduction in HRV in each patient.

Respiratory frequency was obtained by averaging the frequency of respiratory peak of eight sequential HRV measurements. The heart rate and mean blood pressure was obtained by averaging IHR and beat-to-beat mean blood pressure for HRV measurements period.

To confirm a normal distribution, log power was calculated by taking the common logarithm of the power spectra of each component and total power. Data are presented as means \pm SD. Log power, normalized unit power, mean blood pressure, respiratory frequency and heart rate were analyzed using repeated measures of anal-
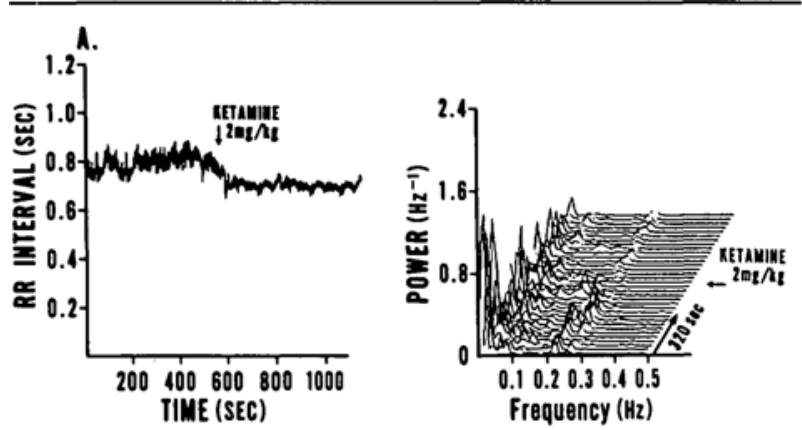

B.
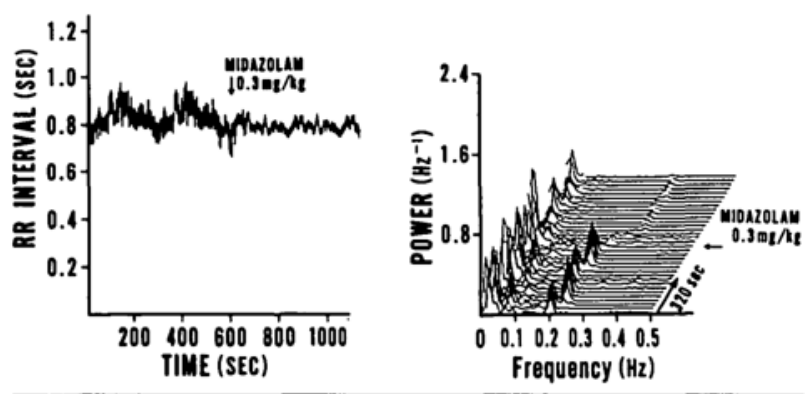

FIGURE 1 Representative RR trendgram (left panel) and power spectral arrays (right panel). Patient A received ketamine and patient B received midazolam. Note the reduction in amplitude of $R R$ trendgram and heart rate variability power at all frequencies after drug administration in both patients. No change in the respiratory frequency after ketamine. Respiratory frequency increased after midazolam.

TABLE I Patient characteristics

\begin{tabular}{lcc}
\hline & Ketamine & Midazolam \\
\hline$n$ & 15 & 15 \\
Sex M & 5 & 6 \\
$\quad$ F & 10 & 9 \\
Age (yr) & $37.6 \pm 11.6$ & $41.6 \pm 11.6$ \\
Weight (kg) & $55.4 \pm 7.9$ & $55.9 \pm 8.3$ \\
Height (cm) & $157.6 \pm 5.9$ & $159.9 \pm 6.7$ \\
\hline
\end{tabular}

Mean \pm SD.

ysis of variance. Differences were considered significant at $P<0.05$.

\section{Results}

Patients of both groups were identical in their demographical characteristics (Table I). There was no difference in the preinduction respiratory rate between the ketamine and midazolam groups. There was no difference in the preinduction and postinduction respiratory rates (Table II) in the ketamine group, but respiratory rate increased in the midazolam group $(P<0.05)$. However, all patients had a respiratory frequency between 0.2 to $0.4 \mathrm{~Hz}$ throughout the study and resultant respiratory variations in HR were within the defined frequency range for our. measurement of HF of HRV. None of the patients showed signs of hypoxia as measured by the pulse oximeter. 
TABLE II Changes in heart rate (HR), mean arterial blood pressure (MAP), and respiratory rate following induction of anaesthesia

\begin{tabular}{lll}
\hline Variables & Ketamine & Midazolam \\
\hline HR (beats ' $\left.\min ^{-1}\right)$ & & \\
- Pre-induction & $77.2 \pm 12.7$ & $75.0 \pm 13.5$ \\
- Post-induction & $94.8 \pm 15.4^{*}$ & $74.6 \pm 15.3$ \\
& & \\
MAP (kPa (mmHg)) & $11.9 \pm 1.3(89.4 \pm 9.4)$ & $12.6 \pm 1.9(94.8 \pm 14.6)$ \\
- Pre-induction & $15.6 \pm 1.6(117.4 \pm 11.7)^{*}$ & $10.2 \pm 1.5(76.7 \pm 11.1)^{*} \dagger$ \\
- Post-induction & & \\
& & \\
Respiratory rate (breaths $\left.\cdot \min ^{-1}\right)$ & $16.0 \pm 3.2$ & $16.5 \pm 2.6$ \\
- Pre-induction & $15.7 \pm 2.2$ & $19.2 \pm 2.4^{*} \dagger$ \\
- Post-induction &
\end{tabular}

$* P<0.05$ vs Pre-induction.

$\dagger P<0.05$ between the groups.

$n=15$, mean $\pm S D$.

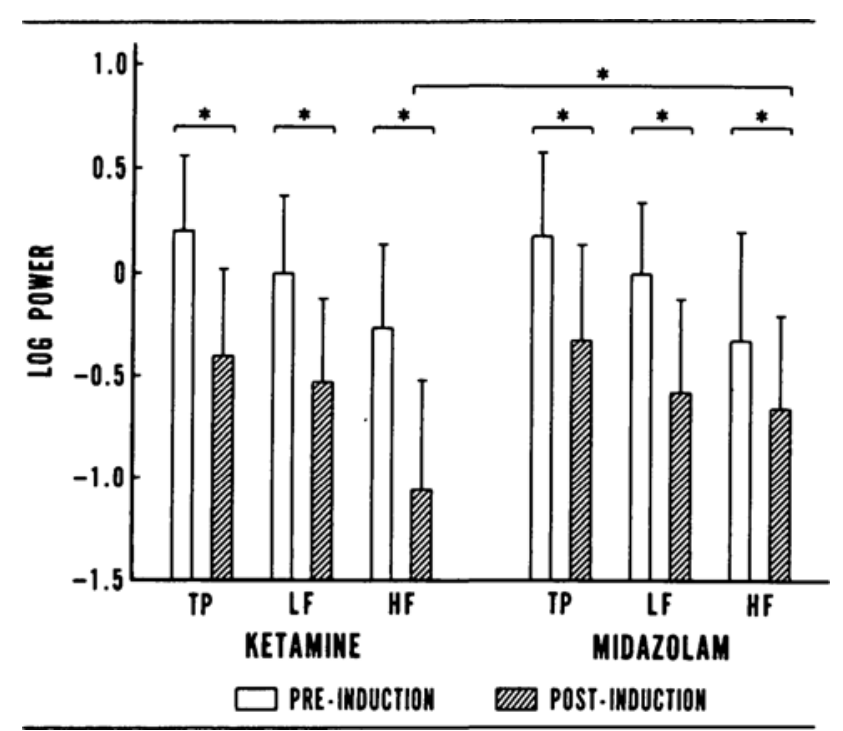

FIGURE 2 Effects of ketamine and midazolam induction on log power of heart rate variability. Bars indicate preinduction versus postinduction power. $n=15$, mean $\pm S D$, *denotes $P<0.05$ between the indicated bars. TP; total power $(0.04-0.5 \mathrm{~Hz}), \mathrm{LF}$; low frequency component (0.04-0.15 Hz), HF; high frequency component (0.15-0.5 $\mathrm{Hz}$ ).

Figure 1 is an example of the $R R$ trendgram and power spectrum changes during ketamine and midazolam anaesthesia. Patient $A$ (top panel) received ketamine, and patient B (bottom panel) received midazolam. The timing of drug administration is noted by an arrow. The RR trendgram shows that both ketamine and midazolam decreased oscillations of RR intervals. Power spectral analysis shows that both ketamine and midazolam decreased the total power along with reduction in power in LF and $\mathrm{HF}$.

Figure 2 shows that effects of ketamine and midazolam

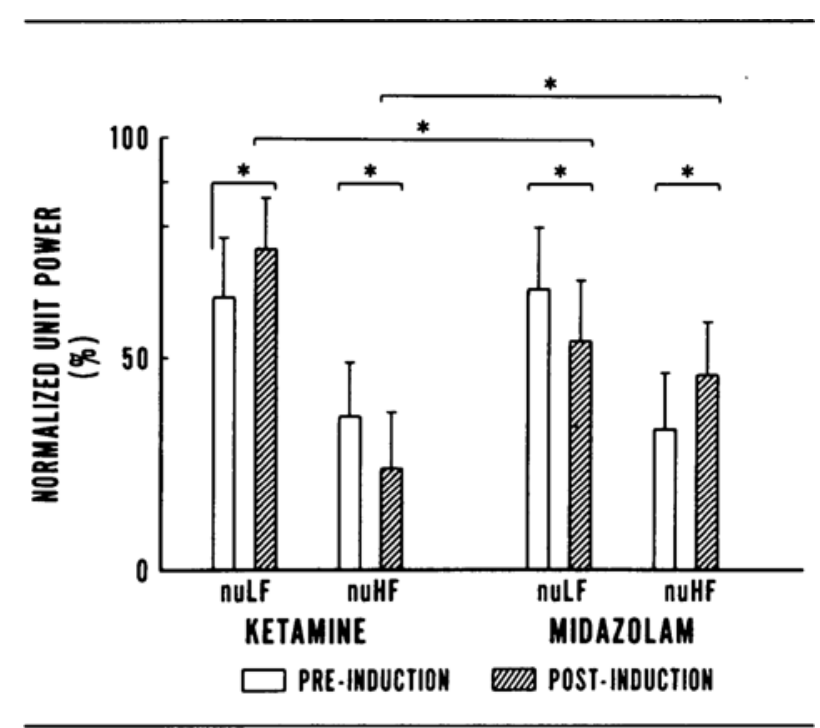

FIGURE 3 Effects of ketamine and midazolam on normalized unit power spectral measurements of heart rate variability. Bars indicate preinduction versus postinduction measurements. $n=15$, mean $\pm S D$, *denote $P<0.05$ between the indicated bars. nuLF; normalized unit low frequency component (low frequency component (LF)/total power (TP) $\times 100 \%$ ), nuHF; normalized unit high frequency component (high frequency component (HF)/total power (TP) $\times 100 \%$ ).

induction on log power of HRV. There were no differences in the preinduction power of TP, LF, and HF between groups. Both, ketamine and midazolam reduced the TP, LF and HF $(P<0.05)$. After induction of anaesthesia, the HF of the ketamine group was less than HF of midazolam group $(P<0.05)$ while there was no difference in LF between the groups.

Figure 3 shows the effects of ketamine and midazolam on normalized unit power spectral measurements of HRV. The nuLF increased from $64 \pm 14 \%$ to $75 \pm 13 \%$ ( $P$ 
$<0.05)$ and nuHF decreased from $36 \pm 14 \%$ to $25 \pm$ $13 \%(P<0.05)$ after ketamine induction. Whereas, the nuLF is reduced from $66 \pm 15 \%$ to $54 \pm 14 \%(P<$ 0.05 ) and the nuHF increased from $34 \pm 15 \%$ to 46 $\pm 14 \%(P<0.05)$ after midazolam.

Mean arterial blood pressure (MAP) and HR increased after ketamine induction $(P<0.05)$, whereas midazolam decreased MAP $(P<0.05)$ without effecting HR (Table II).

Temperature recorded at the end of study was 36.6 $\pm 0.5^{\circ} \mathrm{C}$ in ketamine group and $36.6 \pm 0.6^{\circ} \mathrm{C}$ in midazolam group.

\section{Discussion}

In the present study, the effect of the dose of ketamine or midazolam recommended for induction of anaesthesia on HRV was studied during spontaneous respiration. The results document that both ketamine and midazolam reduced the total power and all frequency components of power in spite of their differential effects on ANS. However, when normalized unit power, which is a fraction of total power, was examined, the nuLF increased and nuHF decreased after ketamine induction. On the contrary, nuLF decreased and nuHF increased after midazolam induction.

Akselrod et al. ${ }^{10}$ and Pomeranz et al. ${ }^{11}$ studied the principal branches of autonomic cardiovascular control and their frequency-specific contribution to HRV. Their study used only power measurements of HRV. They concluded that, in humans, LF of HRV is both sympathetically and parasympathetically mediated, whereas HF is primarily mediated by parasympathetic nervous system activity in the supine position. Recent studies have documented that, under some circumstances of sympathetic activation such as exercise or mental stress, LF is reduced. ${ }^{9,12,13}$ This paradoxical reduction in LF during certain circumstances of increased sympathetic tone apparently stems from the reduction in total power during the experimental intervention. However, examination of the change in the frequency distribution of HRV demonstrated the expected shift toward a greater percentage of power in the low frequency range. ${ }^{9}$ During nitroprusside infusion, when muscle sympathetic activity was increased and mean cardiac parasympathetic activity was diminished, nuLF correlated with sympathetic activity and LF did not. ${ }^{14}$ These studies suggest that under conditions when total power is reduced, changes in LF and HF may not correlate with changes in sympathetic and parasympathetic tone respectively; changes in normalized unit powers of HRV still appear to reflect changes in sympatho-parasympathetic balance. ${ }^{9}$ Because the neural regulation of circulatory function is mainly effected through the interplay of sympathetic and vagal outflow.
Activation of either outflow is accompanied by inhibition of the other, exhibiting a reciprocal relationship. ${ }^{\text {is }}$

In this study we used both power and normalized unit power to assess the ANS activity following induction of anaesthesia with ketamine or midazolam. Ivankovich $e t$ $a l^{16}$ reported cardiostimulant effects of ketamine and showed that ketamine produces sympathomimetic actions via direct stimulation of the central nervous system. In our study, the LF decreased and did not indicate an increase in sympathetic activity after ketamine apart from the fact that HR and MAP increased. However, the nuLF increased and nuHF decreased after ketamine induction. These results indicate that ketamine increases sympathetic activity and shifts the balance of autonomic nervous activity towand the sympathotonic. It has been reported that an induction dose of midazolam decreased plasma norepinephrine concentration suggesting diminution of activity of sympathetic efferent nerve pathway of the reflex. There were reductions in both LF and HF after midazolam induction but the nuLF was reduced with a corresponding increase in the nuHF. This shift in the frequency distribution from the low to the high ranges might reflect sympathetic depression. Thus, quantification of HRV during general anaesthesia may be markedly influenced by the choice of the variables evaluated either by power or normalized unit power. On the basis of our findings, it seems that the normalizing unit procedure should adjust for the reduction in total power of HRV during anaesthesia to assess ANS.

Concerning the reduction in power of HRV using different anaesthesia, we considered three possible mechanisms, central actions of the anaesthetic agents, loss of the spontaneous rhythmical discharges of sympathetic and vagus nerves and respiratory activity.

In patients with episodic increases of intracranical pressure, changes in HRV power paralleled changes in cognitive function such as level of consciousness and orientation. ${ }^{17,18}$ Anaesthetic agents have been suggested to produce unconsciousness by depressing neural activity in the midbrain reticular formation. ${ }^{19}$ One could postulate that the reduction of power of HRV might be caused by the anaesthetic action at CNS depression sites which produce loss of consciousness, because this CNS depression would allow the anaesthetic to produce direct effects on the heart and the peripheral vasculature without compensatory neuronal buffering reflexes. Latson et al. ${ }^{4}$ suggested that loss of consciousness is one of the important mechanisms of the depressant effects of anaesthetics on HRV other than baroreflex depression. Our results obtained in patients who received ketamine or midazolam are in agreement with their hypothesis. Ketamine decreased all frequencies in HRV power despite their sympathetic stimulation and the stimulation of the medulla 
and limbic systems of the brain and simultaneous depression of the cortex.$^{20}$ Midazolam produced little effect on MAP and no alteration in HR. However, marked diminution of total power of HRV was obtained after midazolam administration.

Alternatively, both the cervical sympathetic and the vagus nerves usually have spontaneous rhythmical discharges that are mainly dependent on arterial pulse and respiration and that could affect the HRV. After administration of anaesthetics including ketamine, halothane and barbiturates, sympathetic tone and vagus tone lose the rhythmical discharges and show smaller fluctuations from the baseline. ${ }^{21}$ Thus, loss of the spontaneous discharge of ANS might contribute to the marked reduction of HRV power after anaesthetic administration.

There still remains a possibility that changes in respiratory pattern during anaesthesia may alter the power of HRV. Pagani et al. ${ }^{1}$ observed an increase in vagal tone and simultaneous decrease in sympathetic tone during mechanical ventilation. In this study the lungs were not mechanically ventilated but, one should realize that HF of HRV is both a frequency- and amplitudedependent phenomenon and coincides with respiration. The amplitude of the changes at a given respiratory frequency decreases as the tidal volume decreases and for a given tidal volume, the amplitude decreases as the respiratory frequency increases. ${ }^{22}$ In this study, respiratory frequency increased following midazolam induction and did not change following ketamine induction of anaesthesia. Although tidal volume was not measured, usually tidal volume decreases after induction of anaesthesia. Decreases in respiratory amplitude and changes in respiratory frequency with induction of general anaesthesia may be another important factor in the decreases in total HRV.

In summary, both ketamine and midazolam caused reductions in all frequency components of HRV consistent with previous studies examining changes in HRV during general anaesthesia. These results suggest that a reduction in power of HRV is not only related to a measure of autonomic tone but also depends on the other factors such as loss of consciousness, loss of the spontaneous rhythmical discharges of sympathetic and vagus nerves, and the respiratory effect of the drug. Normalized unit power of HRV, on the other hand, may provide the changes in HRV caused by anaesthetics relevant to their known effects on autonomic activities. We suggest that, when spectral analysis of HRV is used to monitor autonomic activity during anaesthesia, the normalized unit power will be better index for sympathetic and parasympathetic activities than the power.

\section{References}

1 Pagani M, Lombardi F, Guzzetti S, et al. Power spectral analysis of heart rate and arterial pressure variabilities as a marker of sympatho-vagal interaction in man and conscious dog. Circ Res 1986; 59: 178-93.

2 Kato M, Komatsu T, Kimura T, Sugiyama F, Nakashima $K$, Shimada $Y$. Spectral analysis of heart rate variability during isoflurane anesthesia. Anesthesiology 1992; 77: 669-74.

3 Komatsu T, Kimura T, Sanchala V, Shibutani K, Shimada $Y$. Effects of fentanyl-diazepam-pancuronium anesthesia on heart rate variability, a spectral analysis. J Cardiothoracic Vasc Anesth 1992; 6: 444-8.

4 Latson TW, McCarroll SM, Mirhej MA, Hyndman VA, Whitten $C W$, Lipton $J M$. Effects of three anesthetic induction techniques on heart rate variability. J Clin Anesth 1992; 4: 265-76.

5 Scheffer GJ, Ten Voorde BJ, Karemaker JM, Ras HH, De Lange $J J$. Effects of thiopentone, etomidate and propofol on beat-to-beat cardiovascular signals in man. Anaesthesia 1993; 48: 849-55.

6 White PF, Way WL, Trevor AJ. Ketamine - its pharmacology and therapeutic uses. Anesthesiology 1982; 56: 119-36.

7 Marty. J, Gauzit R, Lefevre P, et al. Effects of diazepam and midazolam on baroreflex control of heart rate and on sympathetic activity in humans. Anesth Analg 1986; 65: 113-9.

8 de Boer RW, Karemaker JM, Strackee J. Description of heart-rate variability data in accordance with a physiological model for the genesis of heartbeats. Psychophysiology 1985; 22: 147-55.

9 Malliani A, Pagani M, Lombardi F, Cenutti S. Cardiovascular neural regulation explored in the frequency domain. Circulation 1991; 84: 482-92.

10 Akselrod S, Gordon D, Madwed JB, Snidman NC, Shannon $D C$, Cohen $R J$. Hemodynamic regulation investigation by spectral analysis. Am J Physiol 1985; 249: H867-H875.

11 Pomeranz B, Macaulay RJB, Caudill MA, et al. Assessment of autonomic function in humans by heart rate spectral analysis. Am J Physiol 1985; 248: H151-H153.

12 Pagani $M$, Somers V, Furlan $R$, et al. Changes in autonomic regulation induced by physical training in mild hypertension. Hypertension 1988; 12: 600-10.

13 Pagani M, Mazzuero G, Ferrari A, et al. Sympathovagal interaction during mental stress. Circulation 1991; 83 (suppl II): II-43-II-51.

14 Saul JP, Rea RF, Eckberg DL, Berger RD, Cohen RJ. Heart rate and muscle sympathetic nerve variability during reflex changes of autonomic activity. Am J Physiol 1990; 258: H713-H721. 
15 Levy MN. Sympathetic-parasympathetic interactions in the heart. Cir Res 1971; 29: 437-45.

16 Ivankovich $A D$, Miletich DJ, Reimann C, Albrecht $R F$, Zahed B. Cardiovascular effects of centrally administered ketamine in goats. Anesth Analg 1974; 53: 924-33.

17 Kero $P$, Antila $K$, Ylitalo V, Valimaiki I. Decreased heart rate variation in decerebration syndrome: quantitative clinical criterion of brain death? Pediatrics 1978; 62: 307-11.

18 Lowensohn $R I$, Weiss $M$, Hon $E H$. Heart-rate variability in brain-damaged adults. Lancet 1977; 192: 626-8.

19 Shimoji $K$, Fujioka H, Fukazawa T, Hashiba M, Maruyama $Y$. Anesthetics and excitatory inhibitory responses of midbrain reticular neurons. Anesthesiology 1984; 61:

151-5.

20 Corssen G, Domino EF. Dissociative anesthesia: further pharmologic studies and first clinical experience with the phencyclidine derivation CI-581. Anesth Analg 1966; 45: 29-40.

21 Yamamura T, Kimura $T$, Furukawa $K$. Effects of halothane, thiamylal, and ketamine on central sympathetic and vagal tone. Anesth Analg 1983; 62: 129-34.

22 Brown TE, Beightol LA, Koh J, Eckberg DL. Important influence of respiration on human $R-R$ interval power spectra is largely ignored. J Appl Physiol 1993; 75: 2310-7. 